\title{
Depression Revealing Primary Sjögren's Syndrome with Neurological Involvement
}

\author{
Salem Bouomrani ${ }^{1,2 *}$, and Ines Masmoudi ${ }^{1,2}$ \\ ${ }^{1}$ Department of Internal medicine, Military Hospital of Gabes, Tunisia \\ ${ }^{2}$ Sfax Faculty of Medicine, University of Sfax, Tunisia
}

*Corresponding author: Salem Bouomrani, Department of Internal medicine, Military Hospital of Gabes, Gabes 6000, Tunisia

\begin{abstract}
Primary Sjögren's syndrome (pSS) is the most frequent connective tissue disease but remains very underdiagnosed. Psychiatric manifestations are classified among the central neurological disorders of pSS, and their prevalence is variously estimated according to the series and the recruitment services: $20-70 \%$ of cases. They may be the predominant manifestations of the disease, but pSS remains an underestimated cause of neuropsychiatric disorders. The inaugural psychiatric presentations of this disease are exceptional and represent a real diagnostic challenge for clinicians. We report an original observation of depression as an initial and isolated manifestation revealing neuro-Sjögren ina 60-year-old woman. Only a few similar sporadic cases were previously reported in the world literature. As rare as it is, this clinical presentation of pSS deserves to be known by any healthcare professional.
\end{abstract}

Keywords: Depression; Primary sjögren's syndrome; Neurological involvement; Neuro-sjögren; Behavior disorders

\section{Introduction}

Primary Sjögren's syndrome (pSS) is an autoimmune disease characterized by lymphocytic infiltration of the exocrine glands and the production of autoantibodies, the most characteristic of which are anti-SSA and anti-SSB antibodies [1,2]. With a prevalence of 0.1$3 \%$ and an incidence of $3.9-5.3$ per 100,000 , pSS is the most frequent connective tissue disease, but remains very underdiagnosed [3]. Systemic extra-glandular manifestations of this disease are rare (10$15 \%$ of cases) but often serious and condition the prognosis $[1,4]$. The most frequent of these disorders including pulmonary, renal, neurological, and digestive $[1,4]$. These systemic manifestations are very polymorphic, heterogeneous, and non-specific, justifying the qualification of this syndrome as a "great masquerader" [5]. The specific neurological involvement of this disease (neuro-Sjögren) is not uncommon but very polymorphic and often under-diagnosed $[3,6]$. Depression, classified among the manifestations of central neuro-Sjögren (involvement of the central nervous system) [6,7] can be seen in 32 to $46 \%$ of cases [8]. The inaugural forms of the disease are exceptional and represent a real diagnostic challenge for clinicians [5]. We report an original observation of depression as an initial and isolated manifestation revealing neuro-Sjögren.

\section{Case Report}

A 60-year-old woman, with no notable pathological history, was explored for sadness of the mood with insomnia and permanent headache evolving for three months. She was initially referred by her family doctor to the psychiatric department where antidepressant treatment was prescribed. No improvement was noted after two months of well-observed anti-depressant therapy at optimal doses. The cerebral computed tomography as well as the basic biological investigations (total blood count, renal, hepatic, and thyroid tests) were without anomalies. The somatic examination at our consultation noted dry skin, a fissured tongue, and a reflex quadripyramidal syndrome. No fever, disturbances of consciousness, skin lesions, lymphadenopathy, and visceromegalies were noted. Respiratory and cardio-circulatory status were preserved. Biology showed an erythrocyte sedimentation rate at $56 \mathrm{mmH} 1$ and polyclonal hypergammaglobulinemia at $24 \mathrm{~g} / \mathrm{l}$ without other abnormalities (hemoglobin, leukocytes, platelets, C-reactive protein, calcemia, creatinine, transaminases, uric acid, muscle enzymes, plasma ionogram, glycemia, lipid parameters, and thyroid hormones). 
The ophthalmological examination revealed bilateral keratoconjunctivitis with positive Schirmer's and a breakup time tests. The labial minor salivary gland biopsy showed focal lymphocytic sialoadenitis with a focus score at 2. The immunological tests objectified positive anti-nuclear, anti-SSA and anti-SSB antibodies. Brain magnetic resonance imaging (MRI) showed multiple lesions of the periventricular deep white matter and centrum ovale, in T2 and FLAIR hypersignal, T1 hyposignal, and enhancing after Gadolinium injection (Figure 1). Lumbar puncture and cerebrospinal fluid analysis were without abnormalities. Thus, the diagnosis of pSS with central neurological damage was retained according to the European classification criteria for Sjögren's syndrome without clinical signs or immunological markers of other connective tissue disease. The anti-depressant treatment was stopped, and the patient was treated with three boli of methylprednisolone $(1 \mathrm{~g} /$ day) followed by oral corticosteroid therapy at a dose of $1 \mathrm{mg} / \mathrm{kg} /$ day for four weeks. The evolution was favorable with disappearance of functional complaints and disappearance of depressive symptoms. The control brain MRI at six months of treatment was normal. Currently, and two years later, the patient is still stable without any psychiatric symptoms.

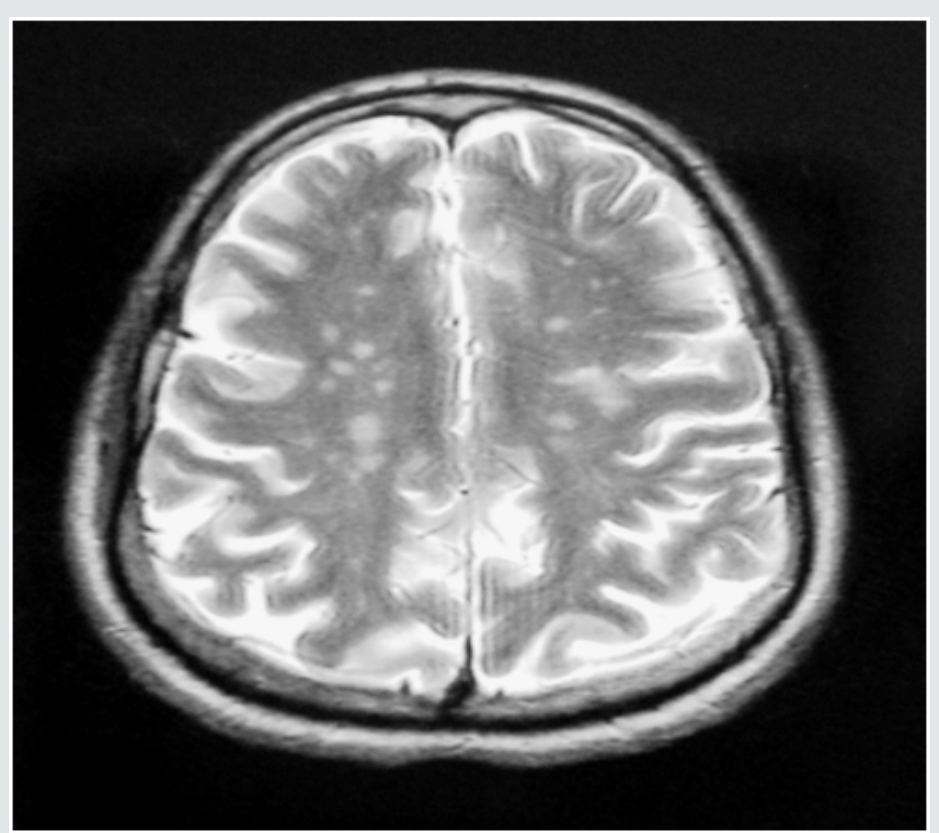

Figure 1: Axial T1-weighted brain MRI with injection: multiple hypersignals of deep periventricular white matter enhancing after Gadolinium.

\section{Discussion}

Neurological manifestations are variously estimated during pSS depending on the series: $8.5-70 \%$ and are by far dominated by peripheral neuropathies [3,6]. The central nervous system involvement is much rarer with very polymorphic clinical aspects $[3,6]$. Psychiatric manifestations are classified among the central neurological disorders of this connective tissue disease, and their prevalence is variously estimated according to the series and the recruitment services: $20-70 \%$ of cases [9]. They may be the predominant manifestations of the disease [9], but pSS remains an underestimated cause of neuropsychiatric disorders [10]. The clinical spectrum of psychiatric manifestations of pSS can include: anxiety disorders, cognitive disorders, attempt to suicide/suicidal ideas, psychotic disorders, auditory and visual hallucinations, agitation/agitated behavior, aggression, paranoia, confusion, emotional lability, depression/depressive disorder, anxiety disorder, sleep disorder/insomnia, delusions, catatonia/catatonic state, schizophrenia/schizophrenia-like symptoms, obsessive- compulsive disorders, other personality disorders, and dementia $[3,6,11,12]$.

The pathophysiology of neuropsychiatric disorders during pSS is not yet fully understood. It appears to be multifactorial involving diffuse central nervous system vasculitis, lymphocytic inflammatory infiltration of nervous tissue, as well as an antibodyantigen reaction between pSS autoantibodies and nerve tissue antigens [6,9]. The exact prevalence of depression during pSS remains unclear [8]. Systematic review and meta-analysis of Cui Y et al, revealed that the prevalence of depression in patients followed for pSS is significantly higher compared to the general population (odds ratio at 5.36, p <0.01). Similarly, the depression scores in pSS's patients are higher than in the control group [8].

The Shen CC et al Nationwide Population-based Retrospective Cohort Study enrolling 2,686 patients with pSS and 10,744 matched controls in Taiwan, objectified significantly higher psychiatric disorders (depressive disorder, anxiety disorder, and sleep disorder) in subjects with pSS compared to healthy controls: 
adjusted hazard risk at $1.829,1.856$, and 1.967 respectfully [13]. Depression associated with pSS is characterized by its partial response or resistance to specific anti-depressant drugs $[3,10,11]$. On the other hand, it usually evolves favorably under corticosteroids [9], and sometimes immunosuppressants [9,12].

More rarely plasmapheresis, intravenous immunoglobulins [12] and even biotherapy have been shown to be necessary to stabilize these neuropsychiatric disorders [3]. Depression as the first manifestation revealing pSS, as in our observation, remains exceptional with only a few sporadic observations $[3,9,10,12]$. It can be seen even in the absence of any specific dry syndrome of the disease [3], be moderate or severe, isolated or associated with other psychiatric disorders $[3,10,12]$, and occur in young people and adolescents making diagnosis more difficult [13]. Finally, it should be noted that depression and in general psychiatric disorders impact negatively the pSS-patient's quality of life and the outcome of their disease $[8,14,15]$.

\section{Conclusion}

As rare as it is, this clinical presentation of pSS deserves to be known by any healthcare professional. The pSS remains an unexpected and under-recognized etiology of neuropsychiatric involvement, especially in young. It should be considered in the differential diagnosis of patients with new psychiatric disorders, even in the absence of sicca symptoms. Systematic neuropsychological tests may contribute to early detection of central nervous system damage during pSS, even in the pre-clinical phase. Only this early diagnosis will improve the prognosis of the disease.

\section{Conflicts of Interest}

None.

\section{References}

1. Patel R, Shahane A (2014) The epidemiology of Sjögren's syndrome. Clin Epidemiol 6: 247-255.
2. Sprecher M, Maurer B, Distler O (2020) Primary Sjögren's Syndrome News on Diagnostics and Therapy. Praxis (Bern 1994) 109(5): 333-339.

3. Hammett EK, Fernandez-Carbonell C, Crayne C, Boneparth A, Cron RQ, et al. (2020) Adolescent Sjogren's syndrome presenting as psychosis: a case series. Pediatr Rheumatol Online J 18(1): 1-15.

4. Demarchi J, Papasidero S, Medina MA, Klajn D, Chaparro Del Moral R, et al. (2017) Primary Sjögren's syndrome: Extraglandular manifestations and hydroxychloroquine therapy. Clin Rheumatol 36(11): 2455-2460.

5. Kumar N, Surendran D, Srinivas BH, Bammigatti C (2019) Primary Sjogren's syndrome: a great masquerader. BMJ Case Rep 12(12): pii: e231802.

6. Perzyńska-Mazan J, Maślińska M, Gasik R (2018) Neurological manifestations of primary Sjögren's syndrome. Reumatologia 56(2): 99105.

7. Margaretten M (2017) Neurologic Manifestations of Primary Sjögren Syndrome. Rheum Dis Clin North Am 43(4): 519-529.

8. Cui Y, Li L, Yin R, Zhao Q Chen S, et al. (2018) Depression in primary Sjögren's syndrome: a systematic review and meta-analysis. Psychol Health Med 23(2): 198-209.

9. Ampélas JF, Wattiaux MJ, Van Amerongen AP (2001) Psychiatric manifestations of lupus erythematosus systemic and Sjogren's syndrome. Encephale 27(6): 588-599.

10. Rosado SN, Silveira V, Reis AI, Gordinho A, Noronha C (2018) Catatonia and Psychosis as Manifestations of Primary Sjögren's Syndrome. Eur J Case Rep Intern Med 5(6): 000855.

11. Lin CE (2016) One patient with Sjogren's syndrome presenting schizophrenia-like symptoms. Neuropsychiatr Dis Treat 12: 661-663.

12. Ong LTC, Galambos G, Brown DA (2017) Primary Sjogren's Syndrome Associated with Treatment-Resistant Obsessive-Compulsive Disorder. Front Psychiatry 8: 100-124.

13. Shen CC, Yang AC, Kuo BI, Tsai SJ (2015) Risk of Psychiatric Disorders Following Primary Sjögren Syndrome: A Nationwide Population-based Retrospective Cohort Study. J Rheumatol 42(7): 1203-1208.

14. Koçer B, Tezcan ME, Batur HZ, Haznedaroğlu Ș, Göker B, et al. (2016) Cognition, depression, fatigue, and quality of life in primary Sjögren's syndrome: correlations. Brain Behav 6(12): e00586.

15. Omma A, Tecer D, Kucuksahin O, Sandikci SC, Yildiz F, et al. (2018) Do the European League Against Rheumatism (EULAR) Sjögren's syndrome outcome measures correlate with impaired quality of life, fatigue, anxiety and depression in primary Sjögren's syndrome? Arch Med Sci 14(4): 830-837.

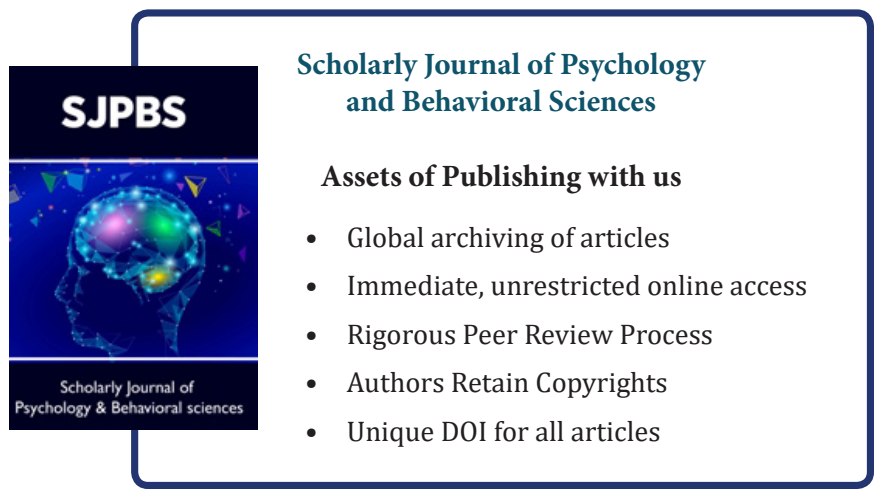

